\title{
Atomistic modelling of large-scale metal film growth fronts
}

\author{
U. Hansen, ${ }^{1}$ P. Vogl, ${ }^{1}$ and Vincenzo Fiorentini ${ }^{1,2}$ \\ 1) Physik-Department and Walter Schottky Institut, Technische Universität München, D-85748 Garching, Germany \\ 2) Istituto Nazionale per la Fisica della Materia and Dipartimento di Fisica, Università di Cagliari, Italy
}

(January 14, 2014)

\begin{abstract}
We present simulations of metallization morphologies under ionized sputter deposition conditions, obtained by a new theoretical approach. By means of molecular dynamics simulations using a carefully designed interaction potential, we analyze the surface adsorption, reflection, and etching reactions taking place during $\mathrm{Al}$ physical vapor deposition, and calculate their relative probability. These probabilities are then employed in a feature-scale cellular-automaton simulator, which produces calculated film morphologies in excellent agreement with scanning-electron-microscopy data on ionized sputter deposition.
\end{abstract}

PACS: 81.10.Aj, 81.15.-z, 68.55.-a

In this Letter we present results of a hierachy of theoretical models developed to describe the growth of metal thin films. Atomic-scale molecular dynamics (MD) and a feature-scale cellular-automaton simulator are combined to yield realistic simulations of film growth during physical vapor metallization of contact vias typical of semiconductor device technology. The MD simulations, accounting in full for the microscopic many-atom dynamics, are used to predict the reaction rates of the processes relevant in physical vapor deposition; the cellular automaton simulator incorporates the reaction rates thus obtained, enabling us to predict and understand the topography of $\mu \mathrm{m}$-scale film fronts, and their relationship to substrate geometry, incident beam energy, and angular beam distribution. Our approach yields a consistent and computationally efficient scheme to predict the topography of metal films on arbitrarily-shaped substrates. A comparison with scanning-electron-microscopy data on sputterdeposited $\mathrm{Al}$-covered trenches demonstrates an excellent level of agreement between theory and experiment.

Our theoretical approach proceeds in three steps, namely (a) a classical interatomic interaction potential for $\mathrm{Al}$ is developed, $(b)$ reaction rates for $\mathrm{Al}$ atoms incident on $\mathrm{Al}$ surfaces are calculated therewith in a $\mathrm{MD}$ simulation, and $(c)$ a cellular automaton is developed and employed to simulate $\mu \mathrm{m}$-scale film fronts, using the reaction rates extracted from MD. In the following, we analyze this procedure and present the relevant results for each step.

Interatomic potential ${ }_{-}$Previously developed classical interatomic potentials 10 for $\mathrm{Al}-\mathrm{Al}$ interactions have mostly focused on bulk and molecular properties. The Ercolessi-Adams potential $\mathbb{l}_{\text {is }}$ a partial exception since, besides reproducing quantitatively the bulk and elastic properties of $\mathrm{Al}$, it also yields interlayer relaxations in good agreement with experiment 1 Using this potential, we obtain surface energies in excellent agreement with $a b$ initio calculations. 6 We thus chose this model as a starting point to develop a new classical many body potential for Al. Our own potential is carefully designed to reproduce the properties of $\mathrm{Al}$ aggregates in a wide range of bonding configurations (from bulk $\mathrm{Al}$ to $\mathrm{Al}$ surfaces and $\mathrm{Al}$ molecules), with special regard to surface properties. Its functionalities significantly extend those of previous embedded atom models, while maintaining a high level of accuracy in reprodycing Al bulk properties and surface formation energies.6 First we introduced an exponential repulsive pair potentiall 10 to account for the short-range interaction of $\mathrm{Al}$ atoms with kinetic energies exceeding 10 $\mathrm{eV}$; this is a key requirement, as the kinetic energies of deposited atoms reach over $150 \mathrm{eV}$ during ionized physical vapor deposition. Second, the embedding function has been readjusted to reproduce observed properties of lowdensity Al structures; after these changes, we obtained a significantly improved agreement of several reference quantities 11 in comparison to $a b$ inito results 1213 and/or experiment 14 Third, we introduced a 5th-order polynomial cutoff function, smoothly cutting off the interaction range of our potential at an interatomic distance of 5.56 $\AA$, slightly larger than third-nearest neighbor distance in bulk Al.

As a further test of our potential, we calculated the barriers for homodiffusion on the low-index faces of fcc Al. Specifically, we considered hopping on $\mathrm{Al}$ (110) along and orthogonal to the [110]-oriented rows, hopping on $\mathrm{Al}$ (100), and concerted exchange on $\mathrm{Al}$ (100). The general level of agreement with ab initio density-functional calculations 12.13 is very good (as can be seen in Table Ii), giving us further confidence in the accuracy of our potential.

TABLE I. Comparison of selected hopping and exchange diffusion barriers on low-index Al surfaces obtained with the present model and in ab initio calculations. $\mathrm{Al}(111)$ is also included for completeness.

\begin{tabular}{lcc}
\hline \hline & This work & Ab initio \\
\hline Al (111) hopping & 0.04 & $0.04^{\mathrm{a}}$ \\
Al (100) hopping & 0.60 & $0.68^{\mathrm{a}}, 0.65^{\mathrm{b}}$ \\
Al (100) exchange & 0.50 & $0.35^{\mathrm{a}}$ \\
$\mathrm{Al}(110) \perp$ hopping & 1.13 & $1.06^{\mathrm{a}}$ \\
$\mathrm{Al}(110) \|$ hopping & 0.30 & $0.60^{\mathrm{a}}$ \\
\hline \hline
\end{tabular}

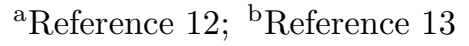


Reaction rates from molecular dynamics - In the second step of our approach, reaction probabilities were calculated in classical molecular dynamics simulations using our $\mathrm{Al}$ interaction potential. In particular we determined, as a function of the energy and off-normal angle of incident $\mathrm{Al}$ atoms, the probability of three processes: adsorption, reflection, and etching (in the latter, the incoming atom's impact on the surface causes the kick-out of one or more substrate atoms). Supercells containing 1320 atoms arranged in 10 atomic layers are employed; cell dimensions are chosen so as to avoid artifacts of the in-plane periodicity. The starting configuration is chosen to be a (111) surface, the one Al surface with the lowest formation energy. All atomic coordinates are allowed to evolve dynamically, except those of the two bottom layers of the supercell. The surface temperature is set at $450 \mathrm{~K}$ (i.e about $1 / 2$ of the melting temperature, and 20 $\%$ larger than the bulk Debye temperature).

We start our simulations with the incident $\mathrm{Al}$ atom placed outside the interaction range of the surface. Its initial kinetic energy is set in the range of 25 to $125 \mathrm{eV}$, and its starting angle off the surface normal in the range $0^{\circ}$ to $60^{\circ}$, which corresponds to typical ionized physical vapor deposition conditions. The trajectories of the incident atom, and of any other atom which may be etched away from the surface upon impact, are then monitored until either a certain time span has elapsed, or the outcoming atoms (in the case of reflection or etching) have traveled a distance of $10 \AA$ away from the surface. Analyzing 200 trajectories per incident energy and angle, we collected a statistically significant sample of well-defined adsorption, reflection, and etching events. The relative probability of the corresponding process is calculated as the ratio of the number of events of each kind to the total number. The typical statistical error in the reaction probabilities thus determined is below $5 \%$.

The behavior of reflection, adsorption, and etch rates as a function of off-normal angle are summarized in Fig. 1 for two representative incident kinetic energies, namely $25 \mathrm{eV}$ in panel (a), and $100 \mathrm{eV}$ in panel (b). At low energies (panel (a)) the adsorption probability (thick solid line, solid squares) decreases from near unity for angles below $20^{\circ}$ to approximately $1 / 2$ for angles in the range of $60^{\circ}$. This decrease is compensated by a corresponding increase in the reflection probability (dashed line, solid triangles). At low energy, etch processes (thin solid line, solid circles) are negligible at all angles. Increasing the incident kinetic energy to $100 \mathrm{eV}$, we find considerable changes in the relative reaction probabilities. Even at small angles, the reflection probability is non-vanishing; the adsorption probability is correspondingly reduced, and is now below 0.7 at all angles. More interestingly, the etching probability is always non-zero, it reaches a maximum of 0.4 at $50^{\circ}$, then decreases as near-grazing angles are approached. For small deviations from normal incidence, the etch rate initially raises, since the probability of a surface atom to gain momentum directed away from the surface increases when the incoming atom ar- rives at an oblique angle at the surface. At large angles of incidence the etch rate drops because of the competing specular reflection events.

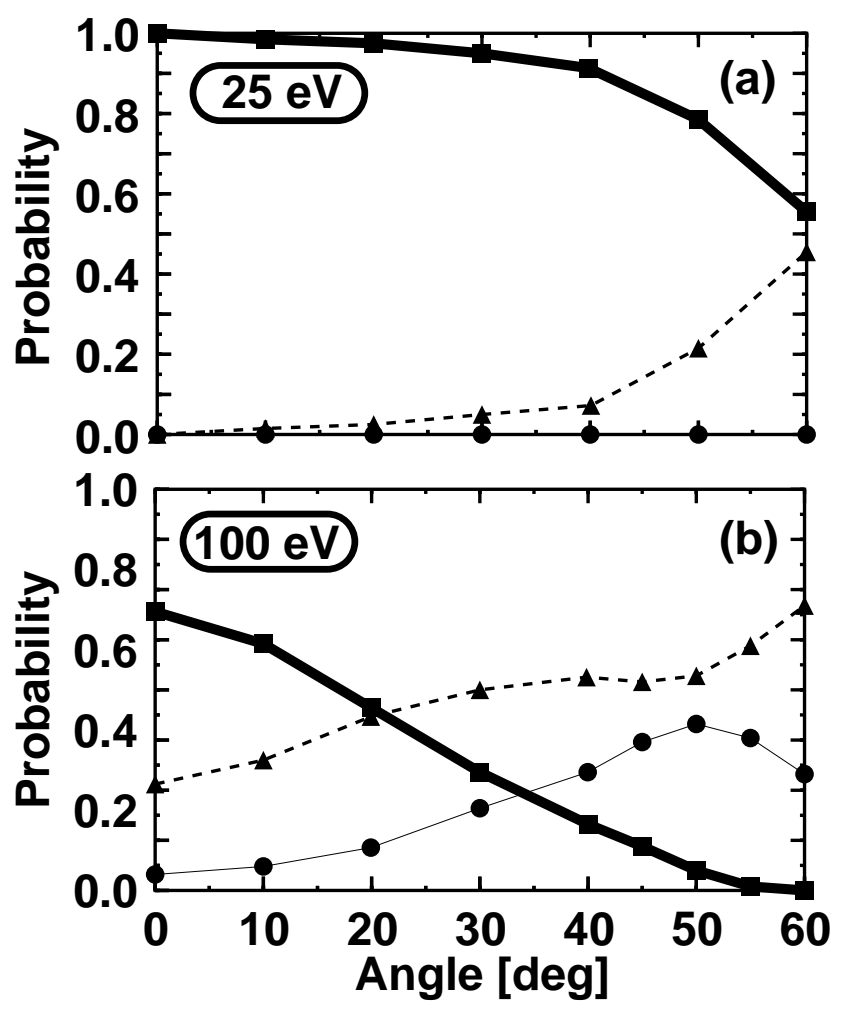

FIG. 1. Reaction probabilities for $\mathrm{Al}$ atoms impinging on $\mathrm{Al}(111)$ with a kinetic energy of $25 \mathrm{eV}$ (panel (a)) and 100 $\mathrm{eV}$ (panel (b)) as a function of the off-normal angle. The processes considered are adsorption (thick solid line, solid squares), reflection (dashed line, solid triangles), and etching (thin solid line, solid circles).

By adjusting the bias voltage between sputter source and deposition target during ionized physical vapor deposition, both the energy regimes just discussed are experimentally accessible. It is expected that they lead to rather different trench topographies, which we simulate with a cellular-automaton technique.

Feature-scale cellular-automaton simulator - In the final step of our approach, we have developed a twodimensional cellular automaton to model the growing film front on a $\mu \mathrm{m}$ scale. The automaton accounts for the effects of flux shadowing, adsorption, reflection, etching, and surface diffusion. The simulated structure is represented in cross section by a two-dimensional grid. Each grid cell represents an $\mathrm{Al}$ atom, and is assumed to have a physical length of $2.5 \AA$ (the effective atomic diameter in Al bulk): thus, for example, a 1- $\mu$-wide structure will be described by 4000 grid cells across.

The atoms are serially and independently emitted from the sputter source far above the surface according to a pre-determined angular and energetic distribution, and move on a straight trajectory, determined also by the 
applied source-target bias, until they strike the growing film front. Interactions in the gas phase are neglected in view of the the low pressure (typically a few tenths of mTorr) and the resulting long mean free path typical of sputter deposition. Spontaneous desorption is also negligible in all the conditions considered.

The impact angle and energy of the atoms hitting the surface determine which process is activated upon impact.15 This may be any one of the three (adsorption, reflection, etching) whose rates have been previously calculated via MD simulations. If the atoms are reflected, or an etch process takes place, the path of the corresponding atoms is further traced until they hit the film surface for a second time. The three basic processes can then take place again, and so forth. Finally, the atoms get either adsorbed, or escape back into the gas phase.

Adsorption present some additional complications. The local diffusion and accomodation mechanism upon adsorption is a key ingredient in deposition models. A reasonable assumption is that incoming atoms are accomodated at a (local) minimum energy site within one diffusion length from the landing site, the diffusion length being determined by the surface temperature and morphology, and the deposition rate. In the cases of interest here, the problem of determining an effective diffusion length for the adsorbed atoms is quite formidable for several reasons. On the one hand, one expects low effective diffusivity due to the very high experimental growth rate (not well controlled, byt in the order of 0.5 $\mu \mathrm{m} /$ minute, or roughly $40 \mathrm{ML} / \mathrm{sec}$ e. 27 ), and also because the growing surface rapidly looses its low-index character becoming essentially disordered. On the other hand, collision energies are large, and (although energy transfer mechanisms on rough surfaces are largely unknown) one may expect multi atom events and transient mobility effects to increase the effective diffusivity. We aim at using parameters that maximize the coordination of the new particle, with the constraint that the impact-to-final site distance is-minimized (so that the local film curvature is minimized 18).

In practice, we set the following criteria for $(a)$ the maximum diffusion distance, and $(b)$ the nature of a minimum-energy site: (a) The maximum diffusion distance is taken to be $d_{\max }=d_{\max }^{\text {thr }}=5$ grid spacings $=12$ Å. All sites within this distance from the landing site are analyzed; the chosen final site is the one closest to the landing site, among those with the highest local coordination (to be defined below, point (b)). Because of this, the effective diffusion length is actually rather smaller than the maximum value $d_{\max }$. Our specific choice of $d_{\max }$ may be simply be regarded as a calibration factor designed to avoid dendritic structures or very flat surfaces which are not observed in the experimental regime of interest here. However, we find a rather sharp change in the dependence of the surface roughness on $d_{\max }$, from rather weak for $d_{\max }>d_{\max }^{\text {thr }}$ to strong for $d_{\max }<d_{\max }^{\text {thr }} .9$ Thus our value $d_{\max }^{\text {thr }}$ is effectively a crossover threshold between the two regimes mentioned. (b) - A minimumenergy site is defined in terms of its local coordination 20 as follows: for each candidate site within the preset maximum distance from the landing site (point $(a)$ ) we calculate the number of atoms contained in a circle center at the candidate site and with a radius of 7 grid spacings. This criterion amounts to selecting the site with maximum average coordination; while similar to that of highest first neighbor coordination, contrary to the latter it avoids pathological choices such as high coordination sites on highly ramified structures.

Results of topography simulations - Figure 2 depicts trench topographies predicted by our model for different deposition conditions, compared to scanning-elestronmicroscope pictures taken in similar conditions. 16 The structure size is $1.2 \mu \mathrm{m}$ across. In the cellular automaton simulation, the emitted atom energies are picked from a Thompson distribution centered at $3 \mathrm{eV}$, as suggested experimentally 12$] 21$ The initial angles of the non-ionized atoms are chosen from a collimated cosine distribution with a maximum off-normal angle of $40^{\circ}$. To mimic the experimental conditions, we assume that $80 \%$ of the emitted $\mathrm{Al}$ atoms get one-fold ionized; for these atoms, the trajectory and impact energy change according to the applied source-target bias.
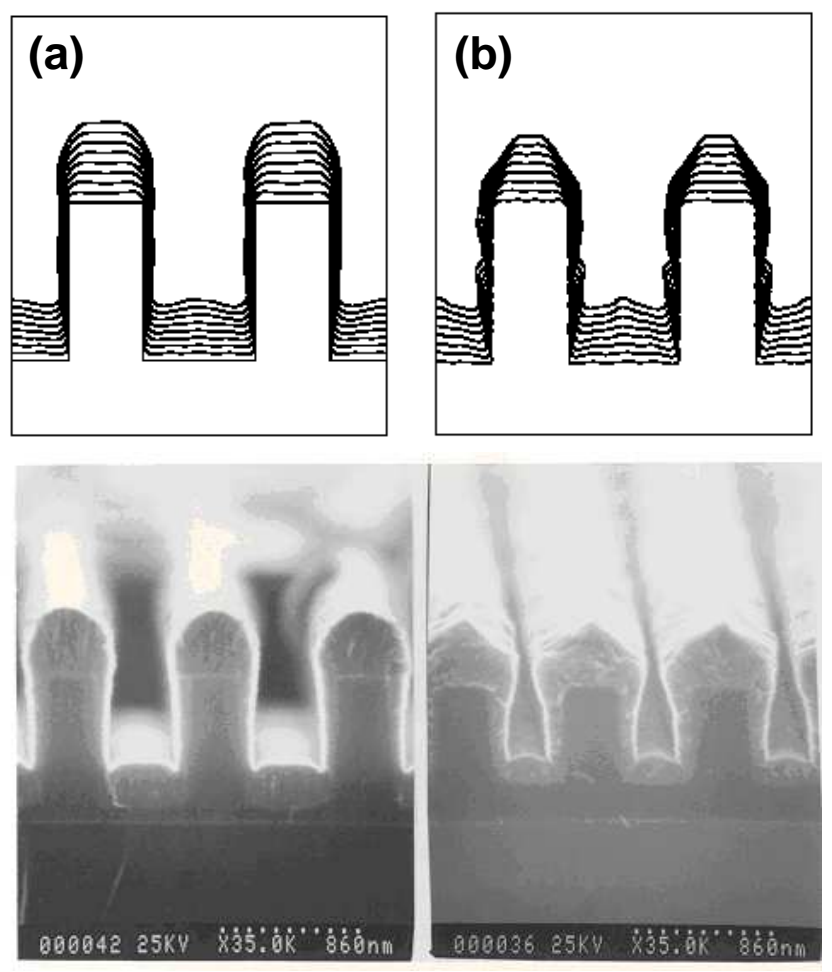

FIG. 2. Film morphologies on trench structures predicted for different ionized gnetron sputtering conditions compared to experiments. 16 In panel (a) we set $80 \%$ ionization, and $10 \mathrm{~V}$ bias, in panel (b) $80 \%$ ionization, and $80 \mathrm{~V}$ bias. Below, a SEM micrograph with experimental results. 
We now describe our results for different values of the the sputter source-to-target bias corresponding to the low and high energy regimes identified in our MD calculations. The results in panel (a) of Fig. 2 were obtained setting the bias to $10 \mathrm{eV}$. In agreement with experiment (also displayed in Fig.2), we predict a film growth front of rounded shape on top of the feature and, due to geometric shadowing, a reduced film thickness at the bottom of the trench. The pile-up at the center of the trench is not only of geometric origin, but also partly due to reflections of atoms impinging on the trench sidewalls. In panel (b) of Fig. 22 we report results obtained with a bias of $80 \mathrm{eV}$. Our calculations predict, in accord with experiment, a roof-like structure on top of the feature. This structure is due to the preferential etching at angles of $50^{\circ}$ (see Fig. I (a)) which leads to a lower deposition rate on the roof-like structure. Simulations of struefures scaled down in size by a similarity transformation 22 (i.e. with the same geometry, relative sizes, and aspect ratios) produced very close results, suggesting that the profiles obtained are largely self-similar at these length scales.22

In conclusion, we have demonstrated the viability of accurate simulations of mesoscopic thin film morphology based on atomic-scale simulations. We performed detailed theoretical calculations of the probabilities for surface reactions taking place during ionized physical vapor deposition conditions, and combined these predictions with $\mu \mathrm{m}$-scale cellular-automaton simulations. Our molecular dynamics calculations revealed strongly energy dependent adsorption, reflection, and etch rates (the latter exhibiting a distinct maximum for high incident kinetic energies at $\sim 50^{\circ}$ ). We were able to predict topographies of metal films deposited on trench structures under different ionized physical vapor deposition conditions in remarkable agreement with experiment. Our results represent a major step ahead over garlier thin film growth models based on rate equations 23 which did not incorporate beam-energy-dependent surface reactions on an atomistic scale.

We gratefully acknowledge financial support by Siemens AG. We thank Dr. A. Spitzer and Dr. A. Kersch for valuable guidance throughout the project, and Dr. Paolo Ruggerone for a critical reading. V.F. was supported by the Alexander von Humboldt-Stiftung during his stay at the Walter Schottky Institut.

${ }^{1}$ M. S. Daw and M. I. Baskes, Phys. Rev. Lett. 50, 1285 (1983); Phys. Rev. B 29, 6443 (1984).

2 J. Cai and Y. Y. Ye, Phys. Rev. B 54, 8398 (1996).
${ }^{3}$ R. G. Hoagland, M. S. Daw, S. M. Foiles, and M. I. Baskes, J. Mater. Res. 5, 313 (1990).

${ }^{4}$ M. I. Baskes, Phys. Rev. B 46, 2727 (1992).

${ }^{5}$ J. Mei and J. W. Davenport, Phys. Rev. B 46, 21 (1992).

${ }^{6}$ F. Ercolessi and J. B. Adams, Europhys. Lett. 26, 583 (1994).

${ }^{7}$ G. N. Kamm and G. A. Alers, J. Appl. Phys. 35, 327 (1964).

${ }^{8}$ V. B. Deyirmenjian, V. Heine, M. C. Payne, V. Milman, R. M. Lynden-Bell, and M. W. Finnis, Phys. Rev. B 52, 15191 (1995).

${ }^{9}$ A. A. Abrahamson, Phys. Rev. 178, 178 (1969).

10 This potential is $\mathrm{V}(r)=A \exp (-B r)-C$, with $A=$ $7255.44 \mathrm{eV}, B=4.42085 \AA^{-1}$, and $C=1.04897 \mathrm{eV}$.

11 The binding energy and vibrational energy of $\mathrm{Al}_{2}$, the (111) homodiffusion barrier, the adsorption energy per atom on $\mathrm{Al}$ (111), and the binding energy of $\mathrm{Al}_{2}$ on $\mathrm{Al}$ (111) with respect to isolated adatoms are $-1.4 \mathrm{eV}, 290 \mathrm{~cm}^{-1}, 0.04$ $\mathrm{eV}, 2.42 \mathrm{eV}$, and $0.50 \mathrm{eV}$. They compare very well with the reference values (all $a b$ initio values from Ref. 12 unless otherwise specified) $-1.4 \mathrm{eV}, 284 \mathrm{~cm}^{-1}$ (experimental, Ref. 14), $0.04 \mathrm{eV}, 3.06 \mathrm{eV}$, and $0.58 \mathrm{eV}$. The LDA adsorption energy is larger than ours due to the known LDA overbinding, but the two values agree well when expressed in percentage of the bulk binding energy $(\sim 73 \%)$.

${ }^{12}$ R. Stumpf and M. Scheffler, Phys. Rev. Lett. 72, 254 (1994); Surf. Sci. 307, 501 (1994); Phys. Rev. B 53,4958 (1996).

13 P. J. Feibelman, Phys. Rev. Lett. 65, 729 (1990).

${ }^{14}$ M. F. Cai. T. P. Djugan, and V. E. Bondybey, Chem. Rev. Lett. 155, 430 (1989).

15 The local normal at the impact site is defined as the direction connecting the impact point to the center of mass of the atoms contained in a circle centered on the impact point and with radius of 10 grid spacings.

16 S. Hamaguchi and S. M. Rossnagel, J. Vac. Sci. Technol. B 13, 183 (1995).

${ }^{17}$ W. D. Westwood, in Microelctronic Materials and Processes, R. A. Levy ed. (Kluwer, Dordrecht 1989), p.133.

18 S. K. Dew, T. Smy, and M. J. Brett, IEEE Trans. Electron. Dev. 39, 1599 (1992).

19 This is due in part to the intrinsic asymmetry produced by the choice of the closest highest-coordination site among those within the $d_{\max }$ circle.

${ }^{20}$ It is known (see e.g. M. Methfessel, D. Hennig, and M. Scheffler, Appl. Phys. A 55, 442 (1995), and references therein) that the binding energy of metals in a structure with local coordination $C$ is given semiquantitatively by $E_{\text {bind }}=A C+B \sqrt{C}$, with $A$ and $C$ constants.

${ }^{21}$ E. Dullini, Nucl. Instr. and Meth. B 2, 610 (1984).

22 A. Barabasi and H. E. Stanley, Fractal Concepts in Surface Growth, (Cambridge UP, Cambridge 1995).

${ }^{23}$ L. J. Friedrich, S. K. Dew, M. Brett, and T. Smy, Thin Solid Films 226, 83 (1995); S. S. Winterton, T. Smy, S. K. Dew, and M. J. Brett, J. Appl. Phys. 78, 3572 (1995); L. J. Friedrich, D. S. Gardner, S. K. Dew, M. J. Brett, and T. Smy, J. Vac. Sci. Technol. B 15, 1780 (1997). 